\title{
Adaptive Compressive Sensing-Based Channel Estimation for 5G Massive MIMO Systems
}

\author{
Ravikanth Sivangi, Devineni Sri Phani Kishore
}

\begin{abstract}
MIMO) is a key innovation in a long way off correspondences to get records paces of more than one instances. vast MIMO framework has pretty big variety of reception apparatuses at the base station (BS) worked for overhauling severa clients simultaneously. it is a promising innovation for acknowledgment of immoderatethroughput far off interchanges. titanic MIMO misuses the excessive degree of spatial opportunity with the purpose that it considerably improves the channel limit and power proficiency of the MIMO framework. The massive MIMO frameworks are comprehensively stated as a full-size putting in innovation for fifth era (5G) wireless correspondence frameworks. but, in massive MIMO frameworks, a careful assurance of the channel nation information (CSI)is needed for motivation in the back of feasible sign discovery, asset distribution and beamforming and so forth. those frameworks having an large range of reception apparatuses at the base Stations, clients want to gauge channels which can be associated with severa portions of transmit recieving wires. due to this, pilot overhead seems to be excessive. on this way, acknowledgment of the right channel state statistics with a base pilot overhead can be a tough issue. Reenactment outcomes finished demonstrates that the proposed calculation can swiftly and precisely determine huge MIMO channel of the difficult to understand channel sparsity and with excessive computational productiveness whilst contrasted and distinct beyond calculations.
\end{abstract}

Watchwords: 5G; substantial MIMO; compressive detecting; sparsity flexible; a channel estimation

\section{INTRODUCTION}

In view of Shannon analyzing hypothesis, compressive detecting (CS) resembles a leap beforehand in signal getting ready society. It changed into supplied thru Donoho, Candès, Romberg, and Tao in 2004. They additionally created medical basics of CS. it is usually utilized for the securing of sign which can be inadequate (or) compressible. For scanty signal, entire facts contained within the signal can be spoken to truly with the help of couple of critical elements, even as contrasted with certainly the period of the signal. commonly the organized components of a signal which modifications fast on the equal time as obeying power law, at that point moreover the ones signal are called compressible sign, allude Fig.1.

Revised Manuscript Received on July 18, 2019

Ravikanth Sivangi, Assistant Professor, CVR College of Engineering, Hyderabad, India (Email: sivangi.ravikanth25@gmail.com)

Dr. Devineni Sri Phani Kishore, Professor, K L University, Vijayawada, Andhra Pradesh, India (Email: mail4usri@kluniversity.in)

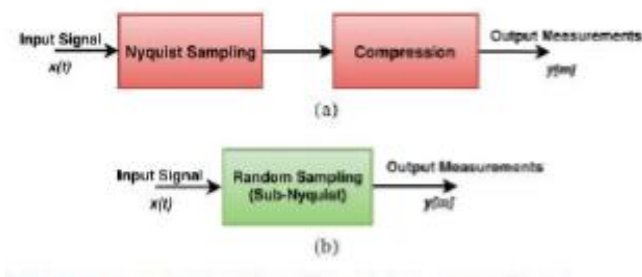

FICURE 2. A comparision of sampling technigates: (a) tracitional sampling, (b) compressive sensing

A sign that's scanty/compressible may be spoken to both in its particular region or in a few change spaces like Fourier alternate, cosine exchange, wavelet trade, and so forth. big MIMO (severa data unique yield) is one of the key innovations applied in reducing aspect bendy cell structures, which has an great range of radio wires at its cell base station. MIMO improves incredibly the channel limit and moreover variety use [1]. In a significant MIMO framework, a selected channel country information (CSI) is pressing with the aim that it affects framework signal popularity, beamforming, asset designation, and so forth. the base station has numerous recieving wires with the goal that which especially develops the multifaceted nature of framework records handling. on this way, in order to make use of the upsides of big MIMO innovation, the more proficient and occasional multifaceted nature channel estimation calculations assumes an vital undertaking. on the element while contrasted with regular MIMO massive MIMO has certainly one of a type advantages. first of all, MIMO has more and more wide variety of reception apparatuses on the BS so straight away precoder is utilized for sign dealing with such MF or ZF. second, having large huge variety of reception apparatuses at BS builds the framework restrict. third, Low electricity advantages inside the uplink/downlink gives the risk to make little cells, which may be both small scale and \%-cells. The huge multi-enter multi-yield (MIMO) innovation makes the restrict of the correspondence framework twofold without increasing the statistics switch capability and transmission manipulate. Channel estimation is the key check in the bodily layer of massive MIMO frameworks. The channel estimation precision affects the framework execution even as the channels are blurring. Channel kingdom data (CSI) is needed to use the terrific benefits of MIMO frameworks $[2,3]$. Be that as it is able to, the accurate CSI is past the vicinity of imagination to assume to find out regularly correspondence structures[4]. because the Base station has grade by grade wide variety of reception apparatuses, the beneficiary wishes to assess increasingly more variety of 
channel coefficients, which builds the frameworks pilot overhead and computational intricacy with the aim that it decreases the throughput of the framework [5]. that is a tough trouble which has been tended to in [3-6]. Writing [13] states that the a few good sized MIMO channels have insufficient attributes so viable channel estimation techniques are possible. a few antique style channel estimation techniques like least-rectangular (LS) calculation , least imply-squared mistake (MMSE) calculation direct least recommend rectangular blunder (LMMSE, and lots of others are carried out even as channel isn't always versatile. but, the genuine channel has a fine multi-meager circumstance. big range of analysts make use of compressive detecting strategies gauge the channel utilizing Pilots. research demonstrates that compressive detecting based totally absolutely channel estimation accomplishes better execution primarily based totally with identical variety of pilots. Compressive detecting based absolutely channel estimation calculations like symmetrical coordinating hobby (OMP) regularization symmetrical coordinating hobby (ROMP) and subspace interest (SP) and so on are in recent times implemented. Be that as it is able to, the above calculations want to foresee the divert sparsity beforehand of time. anyways, the direct sparsity within the true radio correspondence frameworks is generally difficult to understand, with the motive that the above calculations have some limit. The sparsity bendy coordinating interest (SAMP) calculation determine viably sparsity-hard to understand channels, and but the calculation is based upon the iterative advances, which finally ends up inside the quest for elite and higher computational multifaceted nature. giant MIMO frameworks are control a tremendous degree of facts, and the normal compressive channel estimation calculation is tough to regulate between execution estimation and computational multifaceted nature. great MIMO frameworks have sub-channels amongst severa transmitting and accepting reception apparatus sets have the equivalent scanty help set. In flexible and organized subspace interest calculation (ASSP) for mammoth MIMO channel estimation has well ordered method, task meager condition adjustment and the insufficiencies had been perception little of, but the computational multifaceted nature is rather immoderate. This paper gauges sparsityversatile channel us of a information relying at the joint sparsity of a huge MIMO channel. on the off risk that the divert sparsity is obscure in advance of time, a square based totally meager flexible channel estimation calculation is proposed, called (BSAMP). The proposed calculation can be related to the gigantic MIMO channel with the goal that the insufficient features are correctly utilized for higher channel estimation. The important thing aspect right right here is as a long way as possible and assure of the situation of the nonzero additives inside the help set. The pastime effects demonstrate that the proposed strategy can accumulate higher divert estimation in execution and with lower intricacy gave at the same time as the sparsity is difficult to understand.

\section{COMPRESSED MULTIPATH CHANNELS}

Model at the point when the bottom station (BS) in MIMO has $\mathrm{M}$ transmitting reception apparatuses, the part of the arrangement sends symmetrical recurrence department multiplexing (OFDM) indicators, wherein the period of every OFDM signal from every base station recieving twine is $\mathrm{N}$, and $\mathrm{P}(0<\mathrm{P}<\mathrm{N})$ transporters are carried out because the pilot for channel estimation, and the anticipated channel duration L. presently the example for pilot of the ith transmitting recieving wire is $\mathrm{p}(\mathrm{i}), \mathrm{I}=1,2, \ldots, \mathrm{M}$, in which, $\mathrm{p}(\mathrm{i}) \mathrm{np}(\mathrm{j})=\varphi$, If $\mathrm{I} \neq \mathrm{j}$. while channel is transmitted, allow $y(p(i))$ be the pilot sign gotten on the beneficiary., $I=1,2$, ..., M. Disentangling $y(\mathrm{p}(\mathrm{i}))$ as $\mathrm{y}(\mathrm{i})$, currently the channel can be modelled as

$$
y^{(i)}=D^{(i)} F^{(i)} h^{(i)}+m^{(i),} i=1,2, L, M
$$

Where, $D^{(i)}=\operatorname{diag}\{p(i)\}$ is the diagonal array with chosen pilot designs, $\mathrm{m}(\mathrm{i})$ is an added substance white Gaussian Noise with mean worth 0 and fluctuation $\sigma 2, F(i)$ is a $\mathrm{P} \times \mathrm{L}$ sub-lattice of a Fourier coefficients having measurements of $\mathrm{N}$ x $\mathrm{N}$ discrete Fourier change (DFT) grid pilot line components and with the primary $\mathrm{L}$ segments, $\mathrm{h}(\mathrm{i})=$ [h(i)(1), h(i)(2),.., h(i)(L)]T is the motivation reaction of the channel (CIR) relating to the ith radio wire. Presently, accept that $\mathrm{A}(\mathrm{i})=\mathrm{D}(\mathrm{i}) \mathrm{F}(\mathrm{i})$, Then, Equation (1) moves toward becoming

$$
\mathrm{y}^{(\mathrm{i})}=\mathrm{A}^{(\mathrm{i})} \mathrm{h}^{(\mathrm{i})}+\mathrm{m}^{(\mathrm{i}),} \mathrm{i}=1,2, \mathrm{~L}, \mathrm{M}
$$

\subsection{SPARSE ADAPTIVE MATCHING PURSUIT} algorithm

Comprehending the channel estimation relying on compressive detecting is identical as illuminating the accompanying 10 fashionable least issue.

${ }^{\wedge} \mathrm{h}=\arg \min \|\mathrm{h}\|$ zero, problem to $\| \mathrm{y}$-ah $\| \leq \mathrm{C}$

where $\|h\| 0$ is the vector 10 fashionable of the vector $h$ for the non- 0 additives. here the drive response of the channel (h) may be reestablished. what's extra, $\operatorname{spark}(\mathrm{A})$ is the section huge variety in lattice $A$; in spite of the fact that, $2<$ $\operatorname{spark}(\mathrm{A})<\operatorname{rank}(\mathrm{A})+1$. seeing that lattice $\mathrm{An}$ is the $\mathrm{P} X \mathrm{~L}$ fractional Fourier community and $\mathrm{P}<\mathrm{L}$, at that factor, $\|$ h\|zero $<1 / 2(\mathrm{P}+1)$. since the some distance off correspondence channel is scantiness, its most people of the vitality is focused on a couple of taps and a bit piece of the dispersion of strength is beneath the restriction diploma of clamor. The channel length $\mathrm{L}$ is a lot greater prominent than range of faucets. Alongside those lines, with the aid of complete usage of the scanty attributes of the channel, we will utilize modest kind of pilot photographs to get the proper channel estimation; with the motive that range usage is improved. In view of the above end, the amount of nonzero taps inside the channel vector does not surpass ok, and in any occasion L X okay additives are considered as clamor. Presently, gauge the meager scenario and after that find out the additives indoors this range. The assessment between neighboring additives is implemented to select the amount of additives for this cycle and sparsity may be evaluated. The components that with the biggest in contrary evaluation are chosen for the assist set considering that they will the carry channel data. On the point when the notion 
framework fulfills above situations, the scanty signal rebuilding trouble is equal as tackling the arched development trouble. supply An a hazard to be the notion framework and parameter $\mathrm{dk}$ is the base estimation of $\mathrm{d}$ which fulfills the accompanying situation,

$$
(1-\delta)\|\mathrm{h}\| 2<\|\mathrm{Ah}\| 2<\quad(1+\delta)
$$

Where, $\mathrm{h}$ is the sparse signal for $\mathrm{k}$, if $\delta \mathrm{k}<1$, The perception framework A fulfills the kth request RIP. The replica problem ends up following 11 trendy least problem.

${ }^{\wedge} \mathrm{h}=\arg \min \|\mathrm{h}\| 1$, trouble to $\|\mathrm{y}-\mathrm{Ah}\| 2<\mathrm{C}$

2.2. Meager Multipath Channel Estimation

as a result of the joint inadequacy of sizable MIMO channel, the channel vector modified to

$$
\mathrm{w}=\left[\mathrm{w} 1^{\mathrm{T}}, \mathrm{w} 2^{\mathrm{T}}, \ldots \ldots \ldots \ldots \mathrm{wL}^{\mathrm{T}}\right]^{\mathrm{T}}
$$

where wi $=\left[h^{(1)}(i), h^{(2)}(i), \ldots, h^{(M)}(i)\right] T, i=1,2, \ldots, L$, the $\mathrm{i}^{\text {th }}$ sub-block for $\mathrm{w}$. Consider all transmit antennas, the received signal can be written as

$$
\mathrm{z}=\mathrm{Bw}+\mathrm{n}
$$

Where, $\mathrm{B}=\left[\mathrm{B} 1, \mathrm{~B} 2, \ldots, \mathrm{B}_{\mathrm{L}}\right] ; \mathrm{B}_{\mathrm{i}}=\left[\mathrm{a}^{(1)}(\mathrm{i}), \mathrm{a}^{(2)}(\mathrm{i}), \ldots\right.$, $\left.a^{(M)}(i)\right], i=1,2, \ldots, L$ is the ith sub-block of matrix $B, a^{(M)}(i)$ is the $\mathrm{i}^{\text {th }}$ column of the matrix $\mathrm{A}(\mathrm{M})$. If the channel sparsity is unknown then compressed sensing is used to estimate $\mathrm{W}$. Then multiply each sides of above equation via $\mathrm{BH}$, in which $\mathrm{BH}$ is the conjugate transpose of the matrix $\mathrm{B} \cdot \mathrm{B}^{\mathrm{H}} \mathrm{Z}=$ $\mathrm{B}^{\mathrm{H}}(\mathrm{Bw}+\mathrm{n})=\left(\mathrm{I}+\mathrm{B}^{\mathrm{H}} \mathrm{B}-\mathrm{I}\right) \mathrm{w}+\mathrm{B}^{\mathrm{H}} \mathrm{n}=\mathrm{w}+\left(\mathrm{B}^{\mathrm{H}} \mathrm{B}-\mathrm{I}\right) \mathrm{w}+\mathrm{B}^{\mathrm{H}} \mathrm{n}$

Where, I mean ML x ML unit network. On account of the grid $\mathrm{B}$, there is no ideal symmetry condition. BHB - I speaks to the nonzero framework with a little numerical worth. The watched network is $\quad n^{\prime}=\left(\mathrm{B}^{\mathrm{H}} \mathrm{B}-\mathrm{I}\right) \mathrm{w}+\mathrm{B}^{\mathrm{H}} \mathrm{n}$,

Then, above Equation becomes

$$
\mathrm{B}^{\mathrm{H}} \mathrm{Z}=\mathrm{w}+\mathrm{n}
$$

During iteration, Let $\mathrm{R}$ represents an $\mathrm{ML}$ x 1 vector $\mathrm{R}$, where

$$
\mathrm{R}=\left|\mathrm{B}^{\mathrm{H}} \mathrm{r}\right|
$$

Wherein, $r$ speaks to iterative residuals, with introductory well worth $\mathrm{z}$, and components in $\mathrm{BHr}$. presently, the vector $\mathrm{T}$ can be regular as the whole of the squares of each association of $\mathrm{M}$ additives inside the vector. In slight of the addition coefficient of the channel tap is better than the commotion sufficiency, just so for each emphasis of the calculation, The thing amplitudes Ts creates a bigger tempo of progress; and the issue earlier than this function will supply the facts of the channel . along the ones traces, locating the greatest in reverse comparison among contiguous components find out the amount of additives chose on this emphasis, and the components earlier than this role are taken into consideration due to the fact the assist set as bring facts channel . in an effort to decorate the exactness of the selected eleme nts, the regularization way depending on arched advancement want to be acquired. The above examination guarantees that the insufficient estimation of BSAMP calculation first appraises the sparsity furthest restriction via placing the threshold. Meager situation is furthermore evaluated even as finding the most excessive distinction vicinity on this range for detachment of channel faucets and commotion. Contrasted with different stress based completely calculations, the affected components associated with brought substance white Gaussian historical past noise BHn. The BSAMP calculation will considers $\mathrm{BHn}$ and but it considers the vitality scattering because of the non-symmetry of the perception grid (BHB - I) w. The proposed BSAMP calculation use regularization method to channel the components in the assist set for non-obligatory screening, which they're able to improves the exactness of the help set. Alongside these strains, the BSAMP calculation has better estimation execution whilst contrasted with extraordinary cutting-edge calculations. The terrific MIMO framework will recoup the maximum extreme channel measurement, and the sub-channel has consolidated meager situation. The BSAMP calculation makes use of favorable feature of channel rectangular scanty highlights, with the purpose that cycles are decreased. Concurrently there in view of multi problem chose bolster units in each cycle, I t remains far away from the iterative beautify length in order that BSAMP calculation has low computational intricacy.

Tremendous MIMO Channel Estimation based on Compressive Sensing

From the CS hypothesis a sign that's meager in a few area can be recouped from amazingly modest quantity of direct estimations via the use of making use of raised enhancement. This is it recoup specific inadequate vector of higher measurement through bringing right all the way down to its dimension. In some other view the hassle can be considered as estimation of a sign's scanty coefficient as for a assume little of framework. in the beginning the mind of compressive detecting strategies were linked to peculiar detecting frameworks, which might be no adaptive and without delay estimations. Be that as it may, nowadays, the possibility of compressive detecting is through and huge for the most elements supplanted with the aid of meager recuperation. It is probably visible that $\mathrm{hz}, \mathrm{n}$ shows organized sparsity in delay place in which hz,n is $S z, m, n$-inadequate vector because of

$P_{z,}=\operatorname{supp}\left\{\mathrm{h}_{\mathrm{z}, \mathrm{n}}\right\}=\left\{l: h_{z, n}[l]>0\right\}$

With $1 \leq l \leq L$ where $S_{z,}=\left|P_{z, m, n}\right| c$ fulfilling $S_{z, m, n} \ll L$. And due to spatial sparsity we have the following. $P_{z, 1, n}=P_{z, 2, n}=$ $\cdots=P_{z, M, n}$

The required little relationship A dependent on CS hypothesis will be acquired with the goal that the ideal meager recuperation is ensured. It will be additionally demonstrated that any two sections of A have decent cross connection between's them. The proposed pilot configuration is easy to utilize and furthermore simple as far as similarity with current remote systems. For $K$ contiguous OFDM images with indistinguishable example of pilots,

$\mathrm{Y}_{\mathrm{n}}=\mathrm{AnH}_{\mathrm{n}}+\mathrm{W}_{\mathrm{n}}$

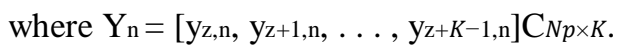

there are numerous calculations that could find out the answer for the problem. The insatiable calculation is a symmetrical coordinating hobby (OMP). For estimation of channel we recommend the SUCoSaMP calculation were given from crucial CoSaMP. There are $\mathrm{Ng}$ sof same parallel managing are required for assessing the massive MIMO channels. there are numerous current strategies

for halting the calculation. at the point while the accompanying ceasing basis fulfilled, if $\|v k+1\| 2>\| v s^{-1 \| 2}$, the emphasis may be halted. The records of correct sparsity 
degree $S z, m, n$ with the aid of and large no longer available and it moreover not basically potential to have in advance getting to know of specific sparsity. The facts about sparsity level assumes a important task in compressive detecting difficulty while explaining underdetermined frameworks. The encouraged SUCoSaM calculation does no longer require in advance data approximately sparsity level because it adaptively gets the sparsity degree. Be that as it may, the proposed SUCoSaMP calculation works similarly because the CoSaMP calculation works. The CoSaMP calculation relies upon on vital OMP. The SUCoSaMP calculation has some wonderful mind with the reason that it ensures that OMP and CoSaMP does no longer deliver and it increment pace of the calculation whilst contrasted with OMP. The upside of SUCoSaMP over OMP, CoSaMP, and one-of-a-kind essential subspace hobby (SP) calculations in that it doesn't require the earlier records of sparsity stage btg66ecause it is a ludicrous supposition. Be that as it can, there are minor contrasts amongst SUCoSaMP and CoSaMP. initially, the SUCoSaMP calculation assesses the channels; 2nd, SUCoSaMP accomplishes adaptively the degree of sparsity. The CoSaMP calculations esimates the inadequate vector without the usage of the organized sparsity degree. The proposed approach stops the cycle with sparsity degree of steady. There are precise types of cycles in SUCoSaMP, one on $\mathrm{k}$ and one on $\mathrm{s}$, in the end the emphasess on $\mathrm{s}$ are halted while $\|v k+1\| 2>\|v s-1\| 2$.

Endeavor consequences

Recreations were performed in MATLAB take a look at gadget if you want to verify the intricacy of the proposed techniques. MSE (suggest square errors) execution of proposed approach is contrasted and cutting-edge OMP, CoSaMP, dependent Subspace Pursuit (SSP), and Adaptive dependent Subspace Pursuit (ASSP) calculations. the bottom station has $1 \times 128$ reception equipment showcase with $M=128$. The proposed framework has switch pace and bearer recurrence are $B=20 \mathrm{MHz}$ and $f c=2 \mathrm{GHz}$, in my opinion. We take into account $N g=$ eight sub-reception apparatus bunches with 16 transmit recieving wires in each collecting. The OFDM subcarriers are taken as $N=2048$, and defend intervening time is $N G=16$ and defer unfold is 6.four $\mu s$, and tweak taken is 16QAM adjustment. The pilot subcarriers are $N p$ in OFDM image which can be from every transmitted reception device of duration L. The multipath diverts are arbitrarily picked wherein channel multipath amplitudes and positions pursue Rayleigh and arbitrary conveyance, in my opinion. discern 1 demonstrates the suggest square error (MSE) execution of the proposed technique SUCoSaMP calculation for numerous estimations of $N p$ versus sign to clamor percentage (SNR). The exhibition of SUCoSaMP is contrasted and the modern-day calculations like OMP and CoSaMP and with SSP and ASSP. From the reenactment results it is tested that the estimation of channel execution of current calculations is superior via increasing range of pilot subcarriers $N p$. The proposed SUCoSaMP calculation plays in a advanced way than each single other calculation because the SUCoSaMP lets in maximum extreme preferred function of sparsity of big MIMO channels. because the sparsity level of massive MIMO in advance facts is obtainable in a sensible a ways off interchanges, the proposed SUCoSaMP calculation

demonstrates the better gain over other every cutting-edge calculation.
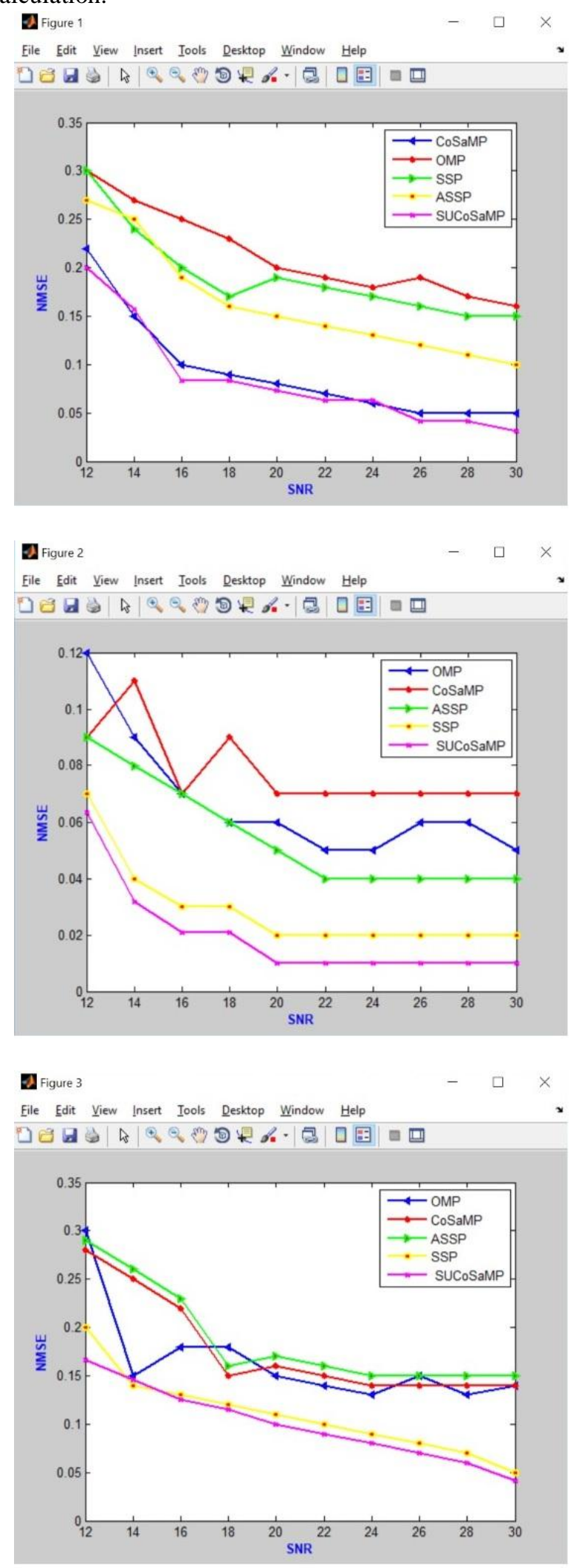

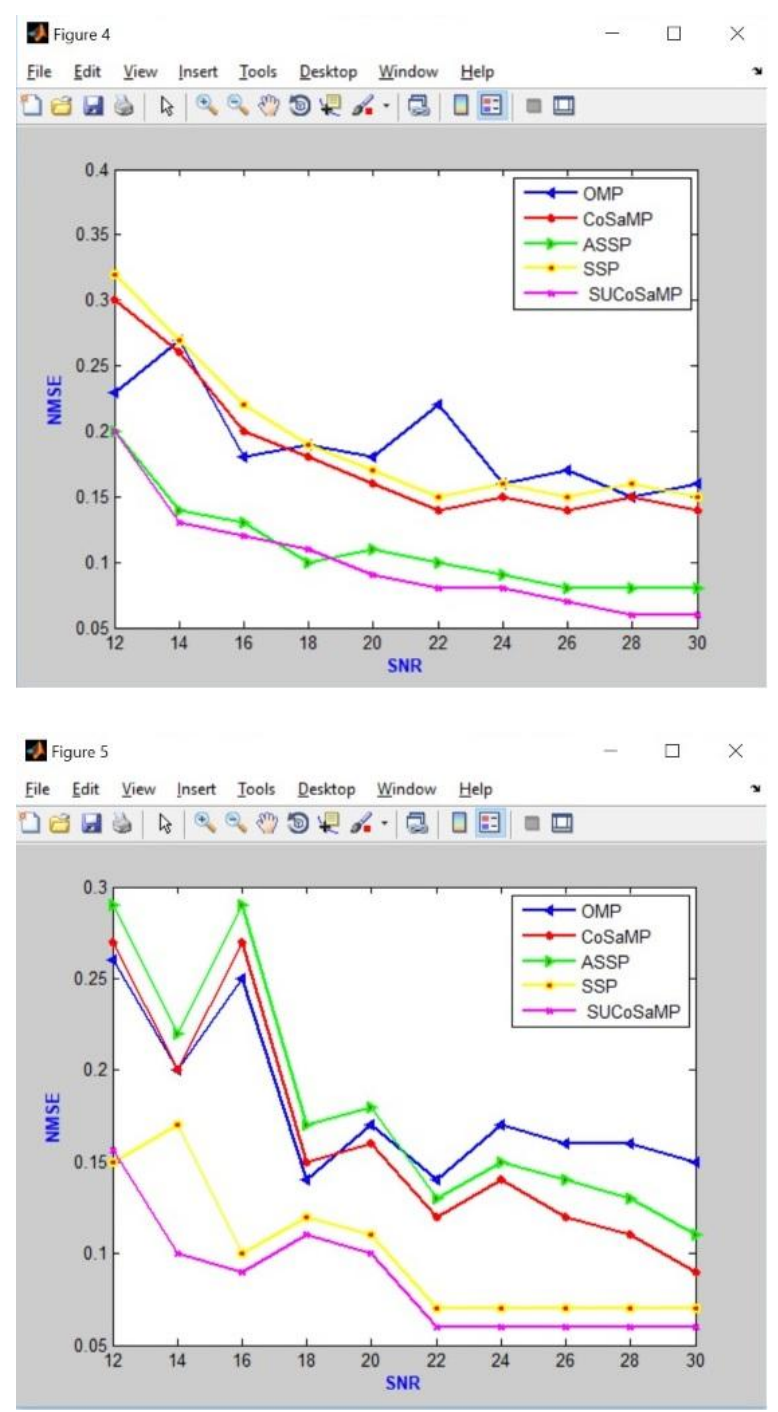

Ends

on this paper we recommend a unique nonorthogonal pilot-prepare method with recognize to Compressive detecting calculation called SUCoSaMP for assessing directs in big MIMO frameworks. From the reenactment results it is hooked up that the pilot overhead is dwindled in MIMO frameworks. With the advantages of spatial and worldly sparsity, proposed nonorthogonal pilot shape technique and estimation of channel plans for the massive MIMO diverts within the defer location, demonstrates that there is decrease in pilot overhead. further, the proposed strategies can be stretched out to multi cells.

\section{REFERENCES}

1. Khan, I.; Zafar, M.; Jan, M.; Lloret, J.; Basheri, M.; Singh, D. Ghostly and energy efficient Low-Overhead Uplink and Downlink Channel Estimation for $5 \mathrm{G}$ big MIMO structures. Entropy 2018

2. Khan, I.; Singh, D. proficient Compressive Sensing based Sarse Channel Estimation for 5G big MIMO structures. AEUE Int. J. Electron. Commun. 2018, 89, 181-a hundred ninety.

3. Kulsoom, F.; Vizziello, A.; Chaudhry, H.N.; Savazzi, P. Pilot decrease techniques for inadequate direct estimation in big MIMO frameworks. In complaints of the 2018 fourteenth Annual conference on wi-fi Onrequest network structures and services (WONS), Isola, France, 6-8 February 2018; pp. 111-116.
4. Liu, L.; Huang, C.; Chi, Y.; Yuen, C.; Guan, Y.L.; Li, Y. Scanty Vector healing: Bernoulli-Gaussian Message Passing. In court cases of the 2017 IEEE global Communications convention, Singapore, 4eight December 2017; pp. 1-6.

5. Huang, C.; Liu, L.; Yuen, C.; solar, S. A LSE and Sparse Message Passing-based totally Channel Estimation for mmWave MIMO systems. In lawsuits of the 2016 IEEE worldwide Communications convention, Washington, DC, united states of america, four-eight December 2016; pp. 1-6.

6. Li, J.; Yuen, C.; Li, D.; Zhang, H.;Wu, X. On half of and half Pilot for Channel Estimation in large MIMO Uplink. 2018. handy at the net: https://arxiv.Org/abs/1608.02112 (were given to on 2 March 2018).

7. Gao, Z.; Dai, L.; Mi, D.; Wang, Z.; Imran, M.A.; Shakir, M.Z. MmWave huge MIMO-primarily based completely far flung backhaul for the 5G extraordinarily-thick set up. IEEE Wirel. Commun. 2015, 22, 13-21.

8. W. Xu, T. Shen, Y. Tian, and Y. Wang, "Compressive channel estimation misusing rectangular sparsity in multi-consumer substantial MIMO frameworks," in lawsuits of the 2017 IEEE wi-fi Communications and Networking convention,WCNC '17, IEEE,2017.

9. Z. Zhou, X. Chen, and D. Guo, "Scanty channel estimation for immense MIMO with 1-piece enter consistent with length," in court casesof the IEEE wireless Communications and Networking convention, WCNC '17, 2017.

10. E. Bjornson and B. Ottersten, "A shape for making equipped based estimation in subjectively linked Rician MIMO channels with Rician aggravation," IEEE Transactions on sign Processing, 2010.

11. X. Mama, F. Yang, S. Liu, and J. Melody, "getting ready succession plan and improvement for prepared compressive sensin based totally direct estimation in vast MIMO frameworks," in proceedings of the GlobecomWork (GC '16), 2016. 